Journal of

Psychopharmacology

\title{
Weight change over two years in people prescribed olanzapine, quetiapine and risperidone in UK primary care. Cohort study in THIN, a UK primary care database.
}

\begin{tabular}{|c|c|}
\hline Journal: & Journal of Psychopharmacology \\
\hline Manuscript ID & JOP-2017-3267.R2 \\
\hline Manuscript Type: & Original Paper \\
\hline Date Submitted by the Author: & 25-Apr-2018 \\
\hline Complete List of Authors: & $\begin{array}{l}\text { Osborn, David; University College London, Division of Psychiatry } \\
\text { Petersen, Irene; University College London, Department of Primary Care } \\
\text { and Population Health } \\
\text { Beckley, Nick; University College London, Department of Primary Care and } \\
\text { Population Health } \\
\text { Walters, Kate; University College London, Department of Primary Care and } \\
\text { Population Health } \\
\text { Nazareth, Irwin; University College London, Department of Primary Care } \\
\text { and Population Health } \\
\text { Hayes, Joseph; University College London, Division of Psychiatry }\end{array}$ \\
\hline $\begin{array}{r}\text { Please list at least } 3 \text { keywords } \\
\text { which relate to your } \\
\text { manuscript:: }\end{array}$ & weight gain, second generation antipsychotics, cohort study, Primary care \\
\hline Abstract: & $\begin{array}{l}\text { Background } \\
\text { Follow-up studies of weight gain related to antipsychotic treatment beyond } \\
\text { a year are limited in number. We compared weight change in the three } \\
\text { most commonly prescribed antipsychotics in a representative UK General } \\
\text { Practice database. } \\
\text { Method } \\
\text { We conducted a cohort study in United Kingdom primary care records of } \\
\text { people newly prescribed olanzapine, quetiapine or risperidone. The primary } \\
\text { outcome was weight in each six month period for two years after treatment } \\
\text { initiation. Weight changes were compared using linear regression, adjusted } \\
\text { for age, baseline weight and diagnosis. } \\
\text { Results } \\
6338 \text { people received olanzapine, } 12984 \text { quetiapine and } 6556 \text { risperidone. } \\
\text { Baseline weight was lowest for men treated with olanzapine ( } 80.8 \mathrm{Kg} \\
\text { versus } 83.5 \mathrm{Kg} \text { quetiapine, } 82.0 \mathrm{Kg} \text { risperidone) and women treated with } \\
\text { olanzapine (67.7Kg versus } 71.5 \mathrm{Kg} \text { quetiapine } 68.4 \mathrm{Kg} \text { risperidone. Weight } \\
\text { gain occurred during treatment with all three drugs. Compared to } \\
\text { risperidone mean weight gain was higher with olanzapine (adjusted co- } \\
\text { efficient }+1.24 \mathrm{~kg} \text { ( } 95 \% \mathrm{CI}: 0.69 \mathrm{~kg}-1.79 \mathrm{~kg} \text { per } 6 \text { months) for men and } \\
+0.77 \mathrm{~kg}(95 \% \mathrm{CI}: 0.29 \mathrm{~kg}-1.24 \mathrm{~kg}) \text { for women). Weight gain with } \\
\text { quetiapine was lower in unadjusted models compared to risperidone, but } \\
\text { this difference was not significant after adjustment. }\end{array}$ \\
\hline
\end{tabular}


Conclusion

Olanzapine is more commonly prescribed to people with lower weight. However, after accounting for baseline weight, age, sex and diagnosis, olanzapine is still associated with greater weight gain over two years than risperidone or quetiapine. Baseline weight does not ameliorate the risks of weight gain associated with antipsychotic medication. Weight gain should be assertively discussed and managed for people prescribed antipsychotics, especially olanzapine.

SCHOLARONE ${ }^{m}$

Manuscripts 


\title{
Weight change over two years in people prescribed olanzapine, quetiapine and risperidone in UK primary care. Cohort study in THIN, a UK primary care database.
}

\author{
David PJ Osborn ${ }^{1,2} \mathrm{PhD}$ \\ Irene Petersen ${ }^{3} \mathrm{PhD}$ \\ Nick Beckley ${ }^{3} \mathrm{PhD}$ \\ Kate Walters ${ }^{3} \mathrm{PhD}$ \\ Irwin Nazareth ${ }^{3} \mathrm{PhD}$ \\ Joseph Hayes ${ }^{1,2} \mathrm{PhD}$
}

1. UCL Division of Psychiatry, UCL, London UK.

2. Camden and Islington NHS Foundation Trust, London UK.

3. Department of Primary Care and Population Health, UCL, London UK

Corresponding author: DPJ Osborn, Division of Psychiatry, University College London, 6th Floor, Maple House, 149 Tottenham Court Road, London W1T 7NF. Email: d.osborn@ucl.ac.uk 
Abstract

Background

Follow-up studies of weight gain related to antipsychotic treatment beyond a year are limited in number. We compared weight change in the three most commonly prescribed antipsychotics in a representative UK General Practice database.

Method

We conducted a cohort study in United Kingdom primary care records of people newly prescribed olanzapine, quetiapine or risperidone. The primary outcome was weight in each six month period for two years after treatment initiation. Weight changes were compared using linear regression, adjusted for age, baseline weight and diagnosis.

Results

6338 people received olanzapine, 12984 quetiapine and 6556 risperidone. Baseline weight was lowest for men treated with olanzapine (80.8 $\mathrm{Kg}$ versus $83.5 \mathrm{Kg}$ quetiapine, $82.0 \mathrm{Kg}$ risperidone) and women treated with olanzapine $(67.7 \mathrm{Kg}$ versus $71.5 \mathrm{Kg}$ quetiapine $68.4 \mathrm{Kg}$ risperidone. Weight gain occured during treatment with all three drugs. Compared to risperidone mean weight gain was higher with olanzapine (adjusted co-efficient $+1.24 \mathrm{~kg}$ (95\% Cl: $0.69 \mathrm{~kg}-1.79 \mathrm{~kg}$ per 6 months) for men and $+0.77 \mathrm{~kg}$ (95\% Cl: $0.29 \mathrm{~kg}-1.24 \mathrm{~kg}$ ) for women). Weight gain with quetiapine was lower in unadjusted models compared to risperidone, but this difference was not significant after adjustment.

Conclusion

Olanzapine is more commonly prescribed to people with lower weight. However, after accounting for baseline weight, age, sex and diagnosis, olanzapine is still associated with greater weight gain over two years than risperidone or quetiapine. Baseline weight does not ameliorate the risks of weight gain 
associated with antipsychotic medication. Weight gain should be assertively discussed and managed for people prescribed antipsychotics, especially olanzapine. 


\section{Introduction}

Weight gain is a common adverse effect of antipsychotic medications, especially in the short term (weeks to months of treatment). People with severe mental illnesses (SMI; such schizophrenia, other non-affective psychotic illnesses and bipolar disorder) who receive these drugs are already at increased risk of obesity, diabetes and cardiovascular disease (de Hert et al.. 2009, Osborn et al. 2007, Osborn et al. 2008). These elevated cardiovascular risks are also reported across broader diagnostic groups such as depression ( Daskalopoulou et al. 2016) and even in people experiencing psychotic symptoms but no SMI diagnosis (Moreno et al., 2013). Antipsychotic agents are currently the first line treatments for control of psychotic symptoms, as well as for relapse prevention. The mixture of adverse effects and therapeutic benefit makes it difficult for the patients and their clinicians to choose the most appropriate drug and dose (Leucht et al., 2013).

The extent of antipsychotic related weight gain in clinical practice over multiple years of treatment is not well understood. There is some trial evidence that antipsychotic related weight gain is more pronounced in the early weeks of treatment, and that people who are underweight or of normal weight are more likely to develop significant weight gain (defined as greater than $7 \%$ of body weight) (Cooper et al., 2016). Younger people may also be more prone to significant antipsychotic related weight gain (Manu et al., 2015). There is less evidence regarding longer term effects. A recent meta-analysis of randomized controlled trials suggested weight gain extended up to 48 weeks after commencing antipsychotics, although few of the studies in the analysis had follow-up periods approaching 48 weeks in duration (Bak et al., 2014). Observational cohorts have found variation in weight change with a range of antipsychotics, but olanzapine (a D2 and 5-HT2 receptor antagonist) is commonly associated with greatest weight gain (Musil et al., 2015). In order that clinicians and patients can make a choice of using an appropriate antipsychotic drug, we need to know the risk of gaining weight in real life clinical 
settings. This will inform preventative strategies against weight gain and associated mortality. Routine clinical data offer an opportunity to describe weight gain in representative clinical samples.

\begin{abstract}
Aims
We aimed to describe recorded weight changes in people receiving their first prescriptions for the three most commonly prescribed antipsychotic medications in the UK namely risperidone (a D2, 5-HT2 and NE alpha-2 receptor antagonist), olanzapine and quetiapine (a D2 and 5-HT2 receptor antagonist and NET reuptake inhibitor) (Marston et al., 2014)
\end{abstract}

Methods

Study design

This was a retrospective cohort study of primary care data recorded between January 1, 2006 and December 31, 2014. We aimed to compare weight in patients receiving one of three antipsychotics as monotherapy and to assess weight change over two years. We included all eligible participants in the follow up to mirror an intention to treat analysis in a trial.

Setting

We used data from The Health Improvement Network (THIN), a large primary care database comprising of electronic medical records from more than 12 million patients across the UK (The Health Improvement Network 2016). General practices that contribute patient data to the THIN network provide anonymised clinical data from patient consultations and administration, generating longitudinal records. Demographic and clinical variables are available the latter are entered as hierarchical Read codes (Chisholm 1990) Townsend score is available as a measure of local social deprivation. Patient populations registered in THIN are representative of the UK population as a whole (Dave and Petersen 


\section{$\underline{\text { Participants }}$}

We included patients within THIN with a new prescription of the three most widely prescribed antipsychotics in the UK: olanzapine, quetiapine or risperidone. If a patient was prescribed one or more of these medications; only their first prescription period was considered (for example, if they had an initial first prescription of olanzapine followed by their first prescription of quetiapine, only their period of time prescribed olanzapine prescription would be included in the study). Patients previously exposed to any antipsychotic medication were excluded from the cohort. Patients may have been prescribed other medications during the study period. We included patients aged between 18 and 99 (inclusive) on the date of their first incident antipsychotic prescription. Patients without a record for sex, age or Townsend Score were excluded, and we only included patients with a minimum of two years of follow up before and after their first prescription of olanzapine, quetiapine or risperidone.

\section{$\underline{\text { Study Variables }}$}

The main outcome of interest was recorded weight over a two year period, compared to their "baseline weight". Baseline weight was defined as the value of the latest weight recording in the two-year period before their incident antipsychotic prescription. Anyone without a weight recording during this two-year period was excluded. Any weight recordings in the two-year period after each patient's incident antipsychotic prescription were extracted with the date of that recording. We also extracted data 
regarding the patients' age, sex, Townsend score (scored 1-5, with increasing value for increasing deprivation level of an area equivalent to 150 households), and record of SMI history. SMI was defined as ever having a record of any of the following: bipolar disorders, schizophrenia like conditions, other psychoses, or being on a practice based SMI register. These were defined according to published Read code lists from previous work (Marston et al., 2014; Osborn et al., 2015).

\section{$\underline{\text { Analysis }}$}

For each of the three drugs, we identified patients who had at least one recording of their weight in the two year period before their incident antipsychotic prescription. For patients with a weight recording after their initial antipsychotic prescription, we calculated the mean of these weight recordings during six-month periods after their incident prescription, namely at 0-6, 6-12, 12-18 and 18-24 months.

We derived stepwise linear regression models with weight difference per 6 months as the outcome variable. We adjusted for age, SMI diagnosis, social deprivation, baseline weight and timing of weight recording (days since incident antipsychotic prescription). The unadjusted model was initially tested with the inclusion of an interaction term for sex, to determine whether subsequent models should be run separately for each sex. All models included a random effect by patient, in order to account for the lack of independence between weight recordings within the same person. Effect estimates were reported with $95 \%$ confidence intervals and $p$-values at the $\alpha=0.05$ cut-off value. We performed an additional analysis excluding people over the age of 65 , who may have different baseline risks for weight change and different indications for antipsychotic treatment. In addition, we calculated the rate of greater than $7 \%$ of baseline weight gain at two years from antipsychotic initiation.

The project received approval from the THIN Scientific Review committee at IMS Health in April 2016. SRC Reference Number: 16THIN013 


\section{$\underline{\text { Results }}$}

We identified 8117, 15653 and 7887 patients with a first prescription of olanzapine, quetiapine and risperidone prescription during the study period. Of these, 6338 (78.1\%), 12984 (82.9\%) and 6556 (83.1\%), respectively, had a weight recording in the two-year period prior to their incident antipsychotic prescription, and were eligible for inclusion in our analysis in terms of having a 'baseline' weight. Of those with missing baseline weight $45.9 \%$ were men and $54.1 \%$ were women, the mean age was 50.9 years (standard deviation 20.5) and $44.4 \%$ had an SMI diagnosis. . Table 1 shows the demographics of the included patients, according to the antipsychotic drug they were prescribed and sex. Baseline weight was lower before prescribing olanzapine, in both men and women (table 1) Over the two year follow-up, the proportions receiving at least one weight record were 5168/6556 (78.8\%) for risperidone, 5777 (91.15\%) for olanzapine and 10378 (79.9\%) for quetiapine.

In the first stage of linear regression, we ran the unadjusted model with and without an additional drugsex interaction term. The likelihood ratio test between these two models found the interaction to be highly significant $(\mathrm{p}<0.001)$, and so all subsequent statistical analysis was stratified by sex.

The mean weight recordings within each of the subsequent six-month periods after first prescription are shown in Figure 1, again by antipsychotic drug and sex.

Weight gain coefficients from the linear regression models are shown in table 2. Townsend score did not significantly improve the performance of either of the fully-adjusted models and was not included in the final models. All models indicated that patients prescribed olanzapine were significantly more likely to put on more weight than patients taking risperidone, with fully-adjusted estimated difference of $+1.24 \mathrm{~kg}$ (95\% Cl: $0.69 \mathrm{~kg}$ to $1.79 \mathrm{~kg}$ ) per six months for men and $+0.77 \mathrm{~kg}$ ( $95 \% \mathrm{Cl}: 0.29 \mathrm{~kg}$ to $1.24 \mathrm{~kg}$ ) per six months for women. The unadjusted models suggested a significant difference in weight change between quetiapine and risperidone users for both men and women but these effects were no longer significant 
in the adjusted models. In the reduced cohort of individuals under the age of 65 , results were similar. In the fully adjusted model, compared to risperidone weight gain in the olanzapine treated group was $+0.94 \mathrm{~kg}(95 \% \mathrm{Cl} 0.50 \mathrm{~kg}$ to $1.38 \mathrm{~kg}$ ) per six months, weight change in the quetiapine treated group was $0.11 \mathrm{~kg}(-0.51 \mathrm{~kg}$ to $0.29 \mathrm{~kg})$ per six months.

By two years, the rate of greater than $7 \%$ weight gain in men was 22.01 (95\% Cl 20.07-24.14) per 100 person-years (pys) for those treated with risperidone, 27.20 (95\% Cl 25.14-29.43) per 100 pys for those treated with olanzapine and 19.14 (95\% Cl 17.79-20.60) per 100 pys for those prescribed quetiapine. In women rates were 24.59 (95\% Cl 22.86-26.44), 29.54 (95\% Cl 27.72-31.48) and $24.33(95 \% \mathrm{Cl} 23.18-$ 25.54) per 100 pys for treatment with risperidone, olanzapine and quetiapine respectively.

\section{Discussion}

Our results show that people receiving all three antipsychotic agents, who have their weight measured, show progressive weight gain over a two year period, running contrary to the belief that weight stabilizes within the short to medium term (Haddad, 2005) and in line with the only other large longitudinal study we are aware of which examines this (Bushe et al., 2012). People prescribed olanzapine had a lower average baseline weight than people prescribed risperidone and quetiapine but they gained more weight in the first six months of treatment and this pattern is continued up to two years after the first prescription. This increased weight gain was not explained by differences in age or sex, and remained true after adjusting for the lower weight of the olanzapine group at baseline, and adjusting for whether someone had a history of SMI. In the supplementary analysis; the proportion of people gaining more than $7 \%$ weight was higher for olanzapine, compared to risperidone.

The strengths of our study include the large representative sample of thousands of people receiving a new prescription of olanzapine risperidone or quetiapine in the UK. The natural design demonstrates 
The limitations include missing data; not every patient has measurements of weight at each of the time points in real life clinical practice, and there was a slight (2-3\%) increased chance for people -in the olanzapine group to have a weight record during the later stages of follow-up, compared to the other two agents. Similar numbers of people had weight recordings in the first six months of follow-up for each drug so the results cannot be fully explained by recording or surveillance bias. However while the figures allow valid comparisons between the agents, we cannot claim that the mean weight gain reflects the true absolute weight gain seen in those who do not have weight recordings. We deliberately kept our analysis simple to describe real life effects. Our analyses are presented using the entire cohorts prescribed olanzapine, risperidone and quetiapine and not stratified by diagnostic group. However we $\underline{\text { did adjust for diagnosis in the multivariate analysis and weight gain with olanzapine remained }}$ significantly higher. The lack of difference in weight gain in the quetiapine treated group, compared to risperidone, might reflect the use of lower doses for non-SMI indications. Future studies might assess the role of antipsychotic dose or duration of treatment, ideally over even longer time periods. They could also explore subgroups who move between different categories of obesity including those who lose significant amounts of weight. However missing data at different time points make this type of analysis challenging in observational data = Indeed, the lower weight gain in the quetiapine treated group might reflect lower doses for non-SMI indications.

Our findings highlight the importance of discussing weight gain with people prescribed any of these three antipsychotic medications, and exploring the risks and benefits of each agent, especially olanzapine. In particular, in order to make informed decisions about their treatment, individuals should be aware of the potential for continued weight gain over at least two years in real life practice. We mimics an intention to treat analysis. 
cannot be certain of the potential for weight gain beyond two years but it is potentially sustained. This discussion should include lifestyle advice and should adhere to the latest guidelines regarding weight gain. Guidelines are available regarding weight gain with antipsychotics and This suggestincludes considering agents such as metformin when weight gain would be a particular concern, or using aripiprazole as an alternative or additional treatment to olanzapine-(Cooper et al., 2016). Our results also highlight the importance of monitoring weight during treatment with antipsychotics as well as other possible sequelae of antipsychotic treatment and weight gain such as disturbances in glucose and lipid metabolism.

\section{References}

Bak M, Fransen A, Janssen J, van Os J, Drukker M (2014) Almost All Antipsychotics Result in Weight Gain: A Meta-Analysis. PLoS ONE 9(4): e94112.

Blak BT, Thompson M, Dattani H, et al. . Generalisability of The Health Improvement Network (THIN) database: demographics, chronic disease prevalence and mortality rates. Inform Prim Care 2011;19:251-5

Bushe CJ, Slooff CJ, Haddad PM, Karagianis JL. Weight change from 3-year observational data: findings from the worldwide schizophrenia outpatient health outcomes database. The Journal of clinical psychiatry. 2012 Jun;73(6):e749-55.Chisholm J. The Read clinical classification. Br Med J. 1990 Apr 28;300(6732):1092

Cooper, S. J., Reynolds, G. P., Barnes, T. R. E., England, E., Haddad, P. M., Heald, A., . . Smith, J. (2016). BAP guidelines on the management of weight gain, metabolic disturbances and cardiovascular risk associated with psychosis and antipsychotic drug treatment. journal of psychopharmacology, 30 (8), 717-748.

Daskalopoulou, M., George, J., Walters, K., Osborn, D. P., Batty, G. D., Stogiannis, D., . . Hemingway, H. (2016). Depression as a Risk Factor for the Initial Presentation of Twelve Cardiac, Cerebrovascular, and Peripheral Arterial Diseases: Data Linkage Study of 1.9 Million Women and Men. PLOS ONE, 11 (4), ARTN e0153838. doi:10.1371/journal.pone.0153838 
Davé S, Petersen I. Creating medical and drug code lists to identify cases in primary care databases. Pharmacoepidemiol Drug Saf. 2009;18(8):704-7.

De Hert M, Dekker JM, Wood D, et al.. “Cardiovascular disease and diabetes in people with severe mental illness position statement from the European Psychiatric Association (EPA), supported by the European Association for the Study of Diabetes (EASD) and the European Society of Cardiology (ESC)". European Psychiatry. 2009; 24: 412-24.

Haddad P. (2005) Weight Changes with atypical antipsychotics in the treatment of schizophrenia. journal of psychopharmacology, 19 (6) 16-27.

Horsfall L, Walters K, Petersen I. Identifying periods of acceptable computer usage in primary care research databases. Pharmacoepidemiol Drug Saf. 2013;22(1):64-9.

In Practice Systems Ltd. The Health Improvement Network (THIN). 2016;

http://www.inps.co.uk/vision/health-improvement-network-thin. (Accessed 24 April 2018).

Leucht S, Cipriani A, Spineli L, et al.. (2013) Comparative efficacy and tolerability of 15 antipsychotic drugs in schizophrenia: a multiple treatments meta-analysis. Lancet 382: 951-962.

Maguire A, Blak BT, Thompson M. The importance of defining periods of complete mortality reporting for research using automated data from primary care. Pharmacoepidemiol Drug Saf. 2009;18(1):76-83.

Manu P, Dima L, Shulman M, Vancampfort D, De Hert M, Correll CU: Weight gain and obesity in schizophrenia: epidemiology, pathobiology, and management. Acta Psychiatrica Scandinavica, 2015.

Marston, L., Nazareth, I., Petersen, I., Walters, K., \& Osborn, D. P. (2014). Prescribing of antipsychotics in UK primary care: a cohort study. BMJ Open, 4 (12), e006135-?. doi:10.1136/bmjopen-2014-006135

Moreno C,Nuevo R,Chatterji S,Verdes E,Arango C,Ayuso-Mateos JL. (2013) Psychotic symptoms are associated with physical health problems independently of a mental disorder diagnosis: results from the WHO World Health Survey. World Psychiatry.12(3):251-7. doi: 10.1002/wps.20070.

Musil R, Obermeier M, Russ P, Hamerle M. Weight gain and antipsychotics: a drug safety review. Expert opinion on drug safety. 2015 Jan 2;14(1):73-96.

Osborn DPJ, Levy G, Nazareth I, Petersen I, Islam A, King M. Relative risk of cardiovascular and cancer mortality in people with severe mental illness from the United Kingdom's General Practice Research Database. Archives of General Psychiatry. 2007; 64; 242-249.

Osborn DPJ, Wright CA, Levy G, King MB, Deo R and Nazareth I. Relative risk of diabetes, dyslipidaemia, hypertension and the metabolic syndrome in people with severe mental illnesses. Systematic review and metaanalysis. BMC Psychiatry 2008; 8:843

Osborn, D. P., Hardoon, S., Omar, R. Z., Holt, R. I., King, M., Larsen, J., . . Petersen, I.

(2015). Cardiovascular risk prediction models for people with severe mental illness: results from the 
prediction and management of cardiovascular risk in people with severe mental illnesses (PRIMROSE) research program. JAMA Psychiatry, 72 (2), 143-151. 


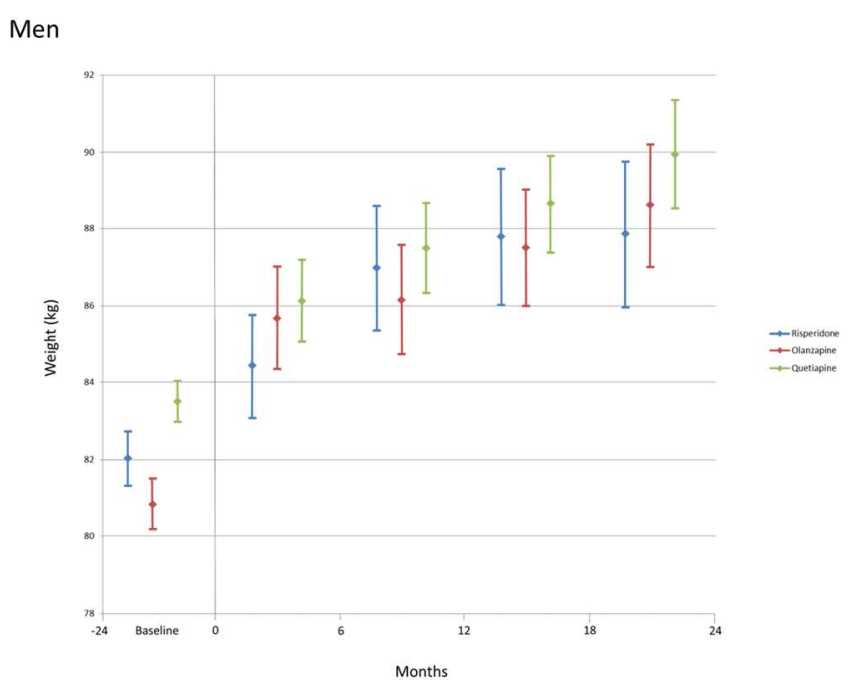

Women

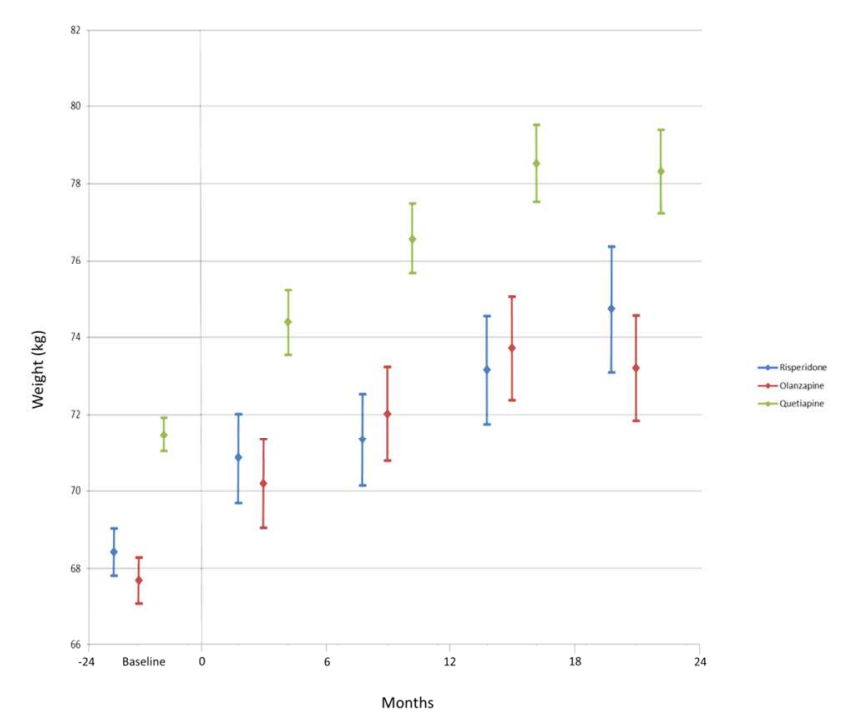

Figure 1: Mean weight recordings $(\mathrm{kg})$ for each of the four six-month periods after patients' incident antipsychotic prescription (olanzapine: red, quetiapine: blue, risperidone: green

$261 \times 437 \mathrm{~mm}(300 \times 300 \mathrm{DPI})$ 
Table 1: Demographics of patients with a baseline weight recording, by antipsychotic drug and sex. Age and baseline weight reported as mean (SD), Townsend score and psychiatric diagnosis as frequency (\%).

\begin{tabular}{|c|c|c|c|c|c|c|}
\hline & \multicolumn{2}{|c|}{ Olanzapine } & \multicolumn{2}{|c|}{ Quetiapine } & \multicolumn{2}{|c|}{ Risperidone } \\
\hline & $\operatorname{Men}(n=2789)$ & Women $(n=3549)$ & Men $(n=5025)$ & Women $(n=7959)$ & Men $(n=2819)$ & Women $(n=3737)$ \\
\hline Age (years) & $48.6(17.8)$ & $53.4(19.3)$ & $57.1(20.3)$ & $55.5(21.6)$ & $56.9(21.3)$ & $62.5(21.9)$ \\
\hline \multicolumn{2}{|c|}{ Baseline weight (kg) 80.8 (18.0) } & $67.7(18.0)$ & $83.5(19.0)$ & $71.5(20.1)$ & $82.0(19.0)$ & $68.4(19.0)$ \\
\hline \multicolumn{7}{|l|}{ Townsend score } \\
\hline 1 & $379(13.6 \%)$ & $638(18.0 \%)$ & $903(18.0 \%)$ & $1368(17.2 \%)$ & 465 (16.5\%) & 704 (18.8\%) \\
\hline 2 & $438(15.7 \%)$ & $667(18.8 \%)$ & $851(16.9 \%)$ & 1345 (16.9\%) & 476 (16.9\%) & $641(17.2 \%)$ \\
\hline 3 & $538(19.3 \%)$ & $697(19.6 \%)$ & $1018(20.3 \%)$ & $1762(22.1 \%)$ & $598(21.2 \%)$ & 786 (21.0\%) \\
\hline 4 & $690(24.7 \%)$ & $824(23.2 \%)$ & $1139(22.7 \%)$ & $1895(23.8 \%)$ & $598(21.2 \%)$ & $849(22.7 \%)$ \\
\hline 5 & $744(26.7 \%)$ & $723(20.4 \%)$ & $1114(22.2 \%)$ & $1589(20.0 \%)$ & $682(24.2 \%)$ & $757(20.3 \%)$ \\
\hline \multicolumn{7}{|l|}{ Diagnosis } \\
\hline SMI & $1044(37.03)$ & $1230(32.91)$ & 1277 (45.79) & $1544(43.51)$ & $1489(29.63)$ & $2464(30.96)$ \\
\hline Schizophrenia & $486(17.24)$ & $419(11.21)$ & 445 (15.96) & 391 (11.02) & 492 (9.79) & $509(6.40)$ \\
\hline Bipolar disorder & $158(5.60)$ & $263(7.04)$ & 341 (12.23) & 574 (16.17) & $510(10.15)$ & 1119 (14.06) \\
\hline Other SMI & $356(12.63)$ & 454 (15.31) & 427 (15.31) & $508(14.31)$ & 366 (7.28) & $581(7.30)$ \\
\hline SMI register & $44(1.56)$ & $94(2.52)$ & $64(2.29)$ & $71(2.00)$ & $121(2.41)$ & 255 (3.20) \\
\hline Non-SMI & 1367 (48.49) & $2145(57.40)$ & 1178 (42.24) & $1733(48.83)$ & 2884 (57.39) & 4897 (61.53) \\
\hline
\end{tabular}




\begin{tabular}{lllllll} 
Anxiety & $446(15.82)$ & $645(17.26)$ & $571(20.47)$ & $746(21.02)$ & $1056(21.01)$ & $1803(22.65)$ \\
Depression & $321(11.39)$ & $567(15.17)$ & $497(17.82)$ & $772(21.75)$ & $860(17.11)$ & $1714(21.54)$ \\
Dementia & $600(21.28)$ & $933(24.97)$ & $110(3.94)$ & $215(6.06)$ & $968(19.26)$ & $1380(17.34)$ \\
No code & $408(14.47)$ & $362(9.69)$ & $334(11.98)$ & $272(7.66)$ & $652(12.98)$ & $598(7.51)$ \\
\hline
\end{tabular}


Table 2. Results from linear regression analysis comparing weight gain on three antipsychotics

\begin{tabular}{|c|c|c|c|c|c|}
\hline & & \multicolumn{4}{|c|}{ Change in weight per 6 month period $(\mathrm{kg})$ compared to risperidone } \\
\hline & & Unadjusted & Age adjusted & Age + SMI adjusted & $\begin{array}{l}\text { Age }+S M I+ \\
\text { baseline weight + half-year } \\
\text { adjusted }\end{array}$ \\
\hline \multirow[b]{2}{*}{ Men } & risperidone & 0 [baseline] & 0 [baseline] & 0 [baseline] & 0 [baseline] \\
\hline & olanzapine & $\begin{array}{l}2.05(95 \% \mathrm{Cl} 1.47 \text { to } 2.63 \\
\mathrm{p}<0.001)\end{array}$ & $\begin{array}{l}1.52(95 \% \mathrm{Cl} 0.95 \text { to } 2.08) \\
p<0.001)\end{array}$ & $\begin{array}{l}1.51(95 \% \mathrm{Cl} 0.94 \text { to } 2.07) \\
p<0.001)\end{array}$ & $\begin{array}{l}1.21(95 \% \mathrm{Cl} 0.66 \text { to } 1.77 \\
p<0.001)\end{array}$ \\
\hline \multirow[b]{2}{*}{ Women } & risperidone & 0 [baseline] & 0 [baseline] & 0 [baseline] & 0 [baseline] \\
\hline & olanzapine & $\begin{array}{l}1.90(95 \% \text { Cl } 1.41 \text { to } 2.39 \\
p<0.001)\end{array}$ & $\begin{array}{l}1.17(95 \% \mathrm{Cl} 0.69 \text { to } 1.65) \text {, } \\
p<0.001)\end{array}$ & $\begin{array}{l}1.16(95 \% \mathrm{Cl} 0.69 \text { to } 1.64, \\
p<0.001)\end{array}$ & $\begin{array}{l}0.79(95 \% \mathrm{Cl} 0.32 \text { to } 1.25 \\
\mathrm{p}=0.001)\end{array}$ \\
\hline
\end{tabular}


Dr Seth

Journal of psychopharmacology

25.4.2018

Dear Dr Seth

Re. Weight change over two years in people prescribed olanzapine, quetiapine and risperidone in UK primary care. Cohort study in THIN, a UK primary care database. (JOP-2017-3267)

Thank you for the opportunity to revise this manuscript again. We have responded to each of the reviewers' points and revised the manuscript with tracked changes.

Many thanks

Yours sincerely

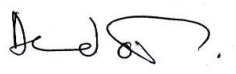

Prof David Osborn

Cover sheet and information:

Title: Weight change over two years in people prescribed olanzapine, quetiapine and risperidone in UK primary care. Cohort study in THIN, a UK primary care database.

\section{Authors and Affiliations:}

David PJ Osborn ${ }^{1,2} \mathrm{PhD}$

Irene Petersen ${ }^{3} \mathrm{PhD}$

Nick Beckley ${ }^{3}$ PhD

Kate Walters ${ }^{3} \mathrm{PhD}$

Irwin Nazareth ${ }^{3} \mathrm{PhD}$

Joseph Hayes ${ }^{1,2} \mathrm{PhD}$

1. UCL Division of Psychiatry, UCL, London UK.

2. Camden and Islington NHS Foundation Trust, London UK.

3. Department of Primary Care and Population Health, UCL, London UK

Corresponding author. Prof Osborn: d.osborn@ucl.ac.uk 


\section{Acknowledgements}

Joseph (F) Hayes and David Osborn are supported by the UCLH NIHR Biomedical Research Centre.

Prof Osborn is also in part supported by the National Institute for Health Research (NIHR) Collaboration for Leadership in Applied Health Research and Care (CLAHRC) North Thames at Bart's Health NHS Trust

This paper summarises independent research funded by the National Institute for Health Research (NIHR) under its Programme Grants for Applied Research (PGFAR) scheme (Grant Reference Number RP-PG-0609-10156). The views expressed are those of the authors and not necessarily those of the sponsor, the NHS, the NIHR or the Department of Health 
